N. E. Piskunov, W. W. Weiss, D. F. Gray, eds.

\title{
Modelling Stellar Magnetoconvection
}

\author{
N.O. Weiss \\ Department of Applied Mathematics $\&$ Theoretical Physics, University \\ of Cambridge, Cambridge CB3 OWA, UK
}

\begin{abstract}
This review focuses on different patterns of magnetoconvection and on aspects of flux expulsion, flux separation and flux pumping. The transition between magnetoconvection and small-scale dynamo action will be described and the rôle of rotation in leading to large-scale dynamo action will be emphasized. An attempt will be made to relate the results of numerical experiments to magnetic features that can be observed on the Sun, and must also be present in other late-type stars.
\end{abstract}

\section{Introduction}

It is now more than half a century since Hannes Alfvén initiated the new subject of magnetohydrodynamics and applied it to the origin of sunspots. That led to studies of the interaction between convection and magnetic fields in stars but the style of these investigations has changed drastically since then. Forty years ago it was only feasible to obtain linear results, relying on analytical techniques. Now it is possible to compute nonlinear solutions for a realistic three-dimensional model of a stellar atmosphere and to compare the results directly with appropriate observations. This progress has led to two contrasting theoretical approaches. One is concerned with predicting quantities that can actually be measured, while the other involves the construction of idealized models that allow different processes to be isolated and explained. The former approach has proved particularly successful in stellar atmospheres, while the latter is more appropriate for stellar interiors (which are anyhow not amenable to direct observation). Reductionist models of magnetoconvection have gradually advanced from kinematic to dynamic calculations, from two to three dimensions and from Boussinesq fluids to fully compressible atmospheres. Nevertheless, despite the advent of supercomputers, it remains impossible to reach the high values of the Reynolds number and the magnetic Reynolds number that prevail in stellar convection zones.

In this survey I shall first review the progress that has been made during the last few decades (cf. Weiss 1991). Much of the stimulus has come from the ever-increasing precision of high-resolution solar observations, starting with the Stratoscope balloon-borne telescope in the 1950s (Danielson 1961) and culminating in the new Swedish Vacuum Solar Telescope on La Palma, whose first results are eagerly awaited. 


\section{Looking back}

Sunspots provided the original motivation for studying the interaction between magnetic fields and convection. The first glimmerings of understanding came in an exchange of letters between Ludwig Biermann, in Germany, and Thomas Cowling, in England, in 1938-39. Biermann suggested magnetic inhibition of convection might explain why spots were cool; Cowling, after characteristically expressing his initial doubts, agreed with this proposal. During the war, Biermann (1941) wrote a survey of stellar convection in which he argued that the magnetic field in a sunspot umbra could suppress convection since the magnetic energy density was much greater than the kinetic energy density of photospheric convection - see Thomas \& Weiss (1992) for the text of his brief statement. The next step was due to Claes Walén: in the course of a rather rambling article (Walén 1949) he mentioned the competition between thermal buoyancy and the Lorentz force produced by the curvature of horizontal field lines, and obtained a criterion for the magnetic suppression of convection which was later resurrected by Cowling (1953).

After this prehistory came the development of linear theory. Cowling (1957) generalised Walén's result and derived a criterion for the transition from undamped oscillations to exponentially growing overturning motion in the absence of any diffusion. Thompson (1951) had already included magnetic and thermal diffusion and showed that if the latter was more effective (as is typically the case in stars) then convection could set in as exponentially growing (overstable) oscillations. Finally, Chandrasekhar $(1952,1961)$ provided the full canonical treatment, with viscosity also included, and distinguished carefully between overstability and monotonically growing instabilities. The next stage came with the development of bifurcation theory and nonlinear dynamics. It was soon found that the initial bifurcation might be subcritical (Veronis 1959; Proctor \& Weiss 1982). Further behaviour was established by expanding about the TakensBogdanov point, with parameters chosen so that the stationary and oscillatory bifurcations coincide (Knobloch \& Proctor 1981; Guckenheimer \& Holmes 1986). The resulting bifurcation patterns could be illustrated in a truncated fifth-order model which also exhibited chaotic oscillations (Knobloch, Weiss \& DaCosta 1981) and Rucklidge $(1992,1994)$ subsequently confirmed that chaos can occur arbitrarily close to the initial bifurcation.

Meanwhile, computers had developed sufficiently to make it possible to carry out numerical experiments and the idealized problem of two-dimensional convection in an electrically conducting, incompressible (Boussinesq) fluid with an imposed vertical magnetic field was systematically investigated (Weiss 1981a, b; Proctor \& Weiss 1982; Weiss 1991). The resulting bifurcation structure could be related to that derived from theory and the nonlinear solutions showed how vigorous convection led to flux expulsion and segregation of the magnetic field from the motion. The next stage was to extend these studies by including compressibility, so that not only stratification but also pressure fluctuations are important (Hughes \& Proctor 1988). The linear theory becomes more complicated, since both fast and slow magnetoacoustic waves are now involved (Weiss 1991), and two-dimensional nonlinear calculations revealed a correspondingly richer variety of behaviour (Hurlburt \& Toomre 1988; Hurlburt et al. 1989; Weiss et al. 1990; Matthews et al. 1993; Proctor et al. 1994). 
By this time the first three-dimensional calculations had appeared, in both the Boussinesq (Arter 1985) and anelastic (Nordlund 1983, 1984) approximations. The latter showed how magnetic flux was swept to the edges of the rising plumes as they impinged on the upper boundary. Thus a magnetic network was formed above the sinking fluid, corresponding to the network that encloses supergranules and, on a much smaller scale, to the concentrations of magnetic flux in intergranular lanes. Since then, as computers have grown more powerful and more sophisticated codes have been developed, simulations have become more realistic and it has become possible to explore parameter ranges that are more extreme.

\section{Detailed simulations}

Numerical modelling of photospheric convection has proved extraordinarily successful (e.g. Spruit, Nordlund \& Title 1990; Stein \& Nordlund 1998). These simulations are fully compressible; they include a realistic equation of state, representing ionization and chemical composition, as well as radiative transfer. The computational domain spans a region that extends from the temperature minimum down to a depth that is greater than the diameter of photospheric granules. Fortunately, behaviour at the photosphere turns out to be remarkably insensitive to the formulation of a lower boundary condition, which inevitably has to be somewhat artificial. The simulated patterns of photospheric convection closely resemble observations of the solar granulation but the calculations are not just restricted to the Sun. Other similar stars can also be modelled and line profiles have been successfully computed. The same approach can naturally be extended to include magnetic fields. Nordlund \& Stein $(1989,1990)$ followed the evolution of the intergranular magnetic network in a facular region, where the mean field is fairly strong, and also attempted to model convection in the umbra of a sunspot. The most recent simulations (Stein, Bercik \& Nordlund 2002) show how magnetic flux is swept first into the intergranular lanes and then concentrated around the larger-scale mesogranules. Small-scale magnetic elements with intense fields appear at corners in this network. Depending on the amount of flux that is involved, they give rise to bright points (slender flux tubes that are laterally heated) or to micropores (somewhat larger concentrations, which appear darker because convective transport is locally suppressed). Since these and other simulations are described elsewhere in these Proceedings by Stein \& Nordlund (2002) and by Vögler \& Schüssler (2002), I shall refrain from discussing them any further here.

\section{Idealized models}

The alternative approach, which follows naturally from the earlier work described in $\S 2$, is to construct more elaborate idealized models, focusing on specific aspects of three-dimensional nonlinear magnetoconvection. In this section I shall describe some recent developments, all of which rely on massive computation. Early two-dimensional kinematic models had already established two related effects, the expulsion of magnetic flux from persistent eddy-like circulations and diamagnetic expulsion by inhomogeneous turbulence (Parker 1979; 

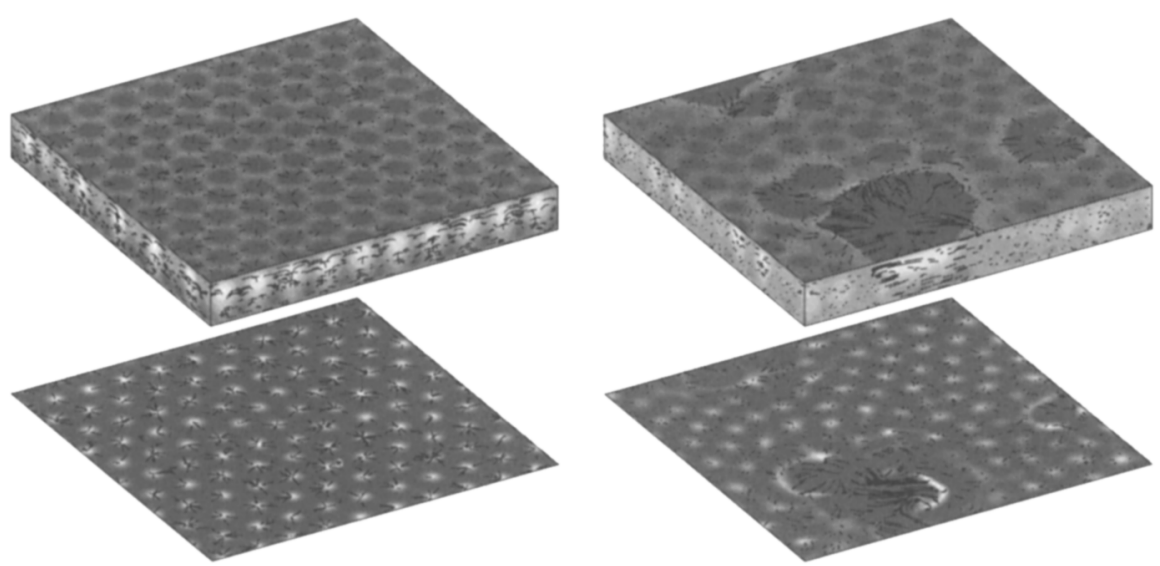

Figure 1. Hysteresis in compressible magnetoconvection for $R=$ 100000 and $Q=2000$. The left-hand panel shows small-scale steady convection in narrow hexagonal cells, enclosed by a magnetic network at the upper surface. The right-hand panel shows a flux-separated solution with a broad and vigorously convecting plume from which magnetic flux has been expelled. The grey-scale shading shows the magnetic field strength at the top and (reflected) the bottom of the layer, with lighter shades indicating stronger fields. Shading of the sidewalls denotes temperature perturbations from the static polytropic solution and the arrows represent the tangential velocity in each surface. (After Weiss et al. 2002.)

Proctor \& Weiss 1982; Zeldovich, Ruzmaikin \& Sokoloff 1983; Mestel 1999), though three-dimensional behaviour proved more complicated to describe (Galloway \& Proctor 1983; Arter 1983). Both of these effects turn out to be important in dynamical modelling of three-dimensional magnetoconvection. The examples I have chosen concern, first, the pattern of convection in the presence of a strong vertical magnetic field, secondly, downward pumping of horizontal flux by compressible convection and, thirdly, the transition from magnetoconvection to small-scale dynamo action in a Boussinesq fluid, which links to the calculations described by Cattaneo (2002) later in these proceedings.

\subsection{Patterns of nonlinear magnetoconvection}

The properties of nonlinear convection in a strongly stratified layer with an imposed vertical magnetic field have been systematically investigated (Weiss et al. 1996; Rucklidge et al. 2000; Weiss, Proctor \& Brownjohn 2002). The fluid is a perfect gas; in the absence of any convection it would be a (strongly superadiabatic) polytrope with a polytropic index $m=1$ and an elevenfold increase in temperature and density across the layer. It is contained in a box with a square cross-section and a ratio of width to height, the aspect ratio $\lambda$, that can be varied. The system is assumed to be periodic in both horizontal directions. At the top and bottom the magnetic field is constrained to be vertical, while the vertical velocity and the tangential stress vanish. The temperature is fixed 

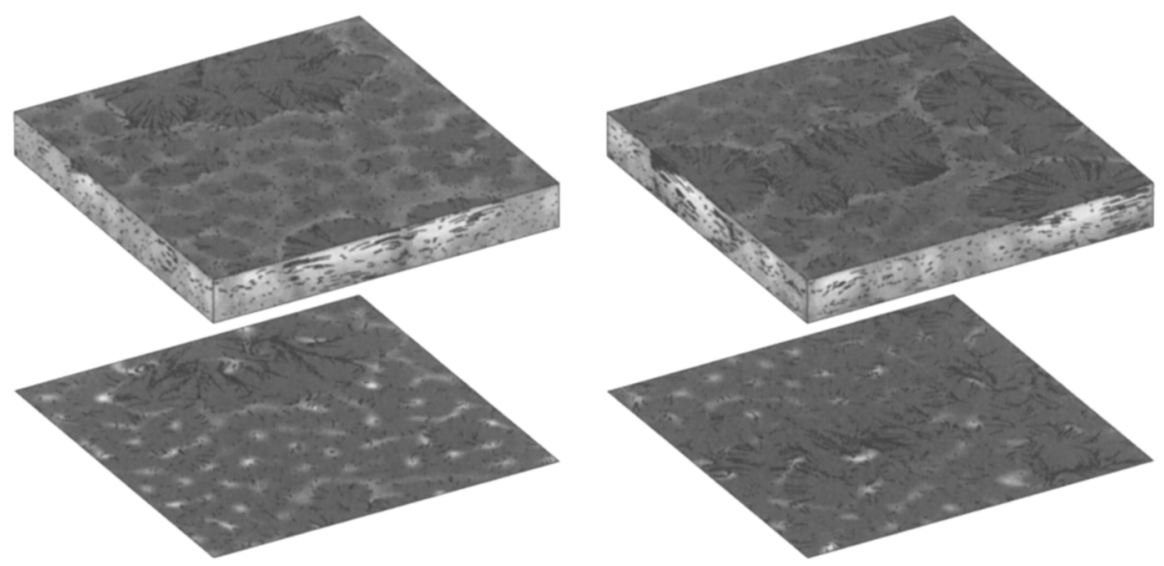

Figure 2. Flux separation in nonlinear magnetoconvection. As Figure 1 but for $Q=1400$ (left panel) and $Q=1000$ (right panel). In both cases only flux-separated solutions are stable and the patterns are time-dependent. The regions of vigorous convection contain clusters of interacting plumes, from which magnetic flux has been expelled. These are surrounded by regions with strong fields and feeble small-scale convection. (After Weiss et al. 2002.)

at the base of the layer; at the top it may either be fixed or else satisfy a 'radiative' boundary condition but this distinction has little effect. The properties of this system depend on five dimensionless parameters. These are the midlayer Rayleigh number, $R$, which measures the superadiabatic gradient, the Chandrasekhar number, $Q$, which is proportional to the square of the imposed field strength, the Prandtl number (the ratio, $\sigma$, of viscous to thermal diffusivity) and the ratio, $\zeta$, of magnetic to thermal diffusivity, plus the aspect ratio, $\lambda$. For the runs to be described here, we set $R=100,000$ and decrease $Q$ from values high enough to halt convection, while $\sigma=1$ and $\zeta$ varies over the range $0.2 \leq \zeta \leq 2.2$ from top to bottom of the layer. This mimics the effects of ionization in the Sun, where $\zeta \ll 1$ at the photosphere, so that oscillatory convection is favoured locally, but $\zeta>1$ at depths greater than $2000 \mathrm{~km}$, favouring steady overturning convection.

Earlier calculations (Weiss et al. 1996) were limited to relatively narrow boxes with $\lambda=2$. They showed a transition from small-scale steady convection in slender hexagonal cells to spatially modulated oscillations (Weiss et al. 1990; Hurlburt, Matthews \& Rucklidge 2000) and then to broad vigorous plumes, as $Q$ was progressively decreased. Once more powerful computing facilities became available it was possible to carry out numerical experiments in wider boxes and it turned out that the patterns of convection had been constrained by choosing such a narrow aspect ratio (Tao et al. 1998b; Rucklidge et al. 2000). In order to avoid this problem it is necessary to set $\lambda \geq 6$ and a systematic survey has now been carried out with $\lambda=8$ (Weiss et al. 2002). When $Q$ is very large the magnetic field is strong enough to suppress convection completely. Linear theory shows that convection sets in at a stationary bifurcation, giving rise to small- 

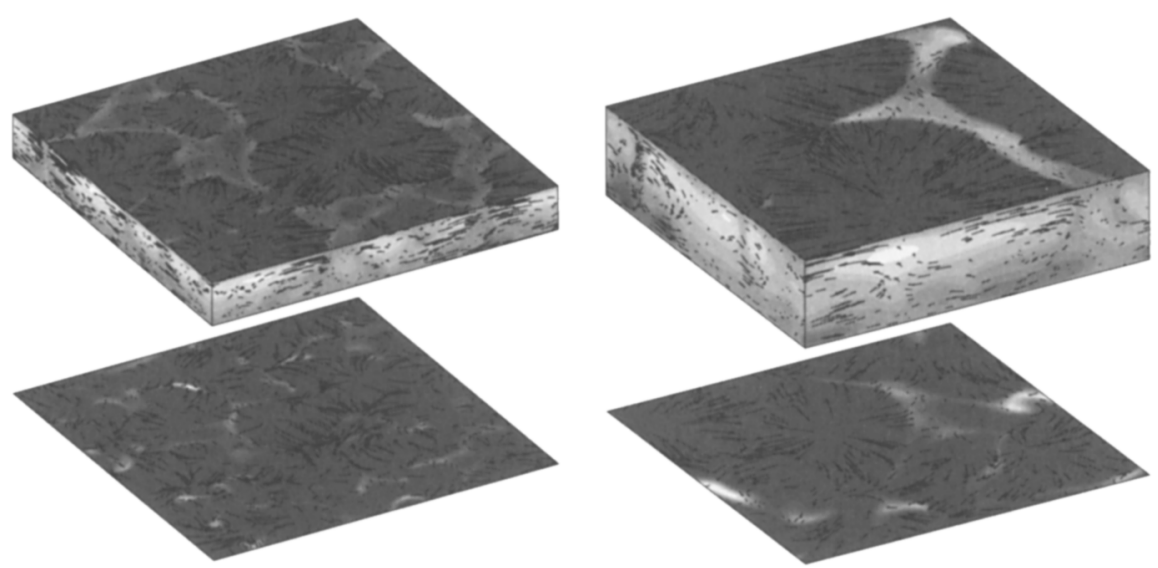

Figure 3. Intermittent fields in the weak field regime. As Figure 1 but for $Q=500$ with $\lambda=8$ (left panel) and, in more detail, $Q=200$ with $\lambda=4$ (right panel). Magnetic flux is squeezed into the narrow lanes that enclose clusters of vigorously interacting plumes. Flux moves rapidly through this network, forming intense but ephemeral fields at the corners. (After Weiss et al. 2002.)

scale steady motion, at $Q \approx 4200$. In the strong field regime $(4000 \geq Q \geq$ 1800 ) we obtain solutions with steady convection in narrow hexagonal cells, corresponding to behaviour in dark nuclei within sunspot umbrae. Figure 1a shows the magnetic field strength at the top and bottom of the box for $Q=2000$. As the rising plumes impinge upon the upper boundary, they sweep magnetic flux aside to form a network that encloses them. Conversely, the (artificial) lower boundary conditions ensure that flux is concentrated beneath these plumes by the converging flow. This pattern remains stationary until $Q=1600$, when it becomes oscillatory. The plumes do not move but they alternate aperiodicically in vigour, giving rise to spatially modulated oscillations.

When $Q=1400$ a totally new pattern appears, as shown in Figure 2a: some of the slender plumes amalgamate, convecting so vigorously that magnetic flux is expelled. This results in flux separation, where strong fields (with weakly convecting narrow cells) are segregated from actively convecting plumes. Moreover there is hysteresis: with suitable initial conditions, flux-separated solutions can be obtained for $Q \leq 2000$, as illustrated in Figure 1b. Flux separation continues as $Q$ is decreased through a regime of moderate field strengths, with $1400 \geq Q \geq 600$. Figure $2 \mathrm{~b}$ shows a typical pattern, for $Q=1000$ : vigorous field-free convection now occupies a much larger fraction of the region, with weaker small-scale convection in the remainder, where the field is strong. As the imposed field is further reduced, magnetic flux is progressively confined to lanes that enclose the clusters of broad and interacting plumes. In the pattern for $Q=500$, shown in Figure 3a, there is still some fine structure in these lanes but by $Q=200$ magnetic flux is confined to regions of strong field in which convection is locally suppressed, as illustrated in the detailed image of Figure $3 \mathrm{~b}$. The flux apparently moves like a magnetic fluid through the network that encloses 
these mesoscale clusters. The strongest fields appear at corners in this network but these intense fields are ephemeral and change as the pattern alters. In this regime no isolated flux tubes can be found. This pattern represents behaviour in plage regions in the photosphere. Computational difficulties have so far prevented us from proceeding to yet lower values of $Q$, corresponding to the quiet Sun.

\subsection{Flux pumping}

The problem of kinematic flux expulsion in steady laminar motion has a venerable history, going back to J.J. Thomson (1893). In particular, an initially horizontal field embedded in a layer of two-dimensional eddies will be expelled into boundary layers at the top and bottom of the layer if the magnetic Reynolds number is sufficiently high (Weiss 1966). Inhomogeneous turbulence may also exhibit a similar diamagnetic effect, pumping magnetic flux down the gradient of turbulent intensity (Zeldovich 1956; Rädler 1968). The latter process has been demonstrated numerically both for convectively driven and for forced turbulence in fully dynamical two-dimensional calculations (Nordlund et al. 1992; Brandenburg et al. 1996; Tao, Proctor \& Weiss 1998a). In a compressible fluid, up-down symmetry is broken and three-dimensional convection leads to the appearance of hexagonal cells. Then there is a topological distinction between the network of sinking fluid and the isolated rising plumes. Drobyshevski \& Yuferev (1974) argued that magnetic flux would therefore be pumped preferentially downwards. It turns out, however, that there is a much more potent mechanism. All simulations of stratified compressible convection show a strong contrast between the gently rising plumes and the sinking fluid, which is focused into rapidly descending plumes at corners in the network (e.g. Spruit et al. 1990). Since magnetic fields 'feel' velocity rather than momentum density through the induction equation, the field is predominantly influenced by these vigorous sinking plumes and is therefore pumped downwards.

Magnetic pumping has been modelled by placing a strongly superadiabatic layer above one that is stably stratified and investigating the fate of an initially horizontal field (Tobias et al. 1998, 2001; Dorch \& Nordlund 2001). The vigorous sinking plumes penetrate into the stably stratified region, exciting internal gravity waves, and are effective in pumping magnetic flux downwards and out of the unstable region. Figure 4 shows the results of a calculation where the lower layer is only weakly stable (Thomas et al. 2002). (In the absence of any motion the two layers would have had polytropic indices $m=1$ and $m=1.75$, where $m=1.5$ for adiabatic stratification.) The volume renderings in this figure show initial and final states of the vertical velocity $w$, of the enstrophy density $\Omega^{2}$ (the square of the vorticity) and of the normalised magnetic energy density $B^{2}$. After a relatively short time, most of the horizontal magnetic flux has been pumped into the stable region, leaving tangled fields behind in the turbulent convecting layer above. The fields themselves are represented in Figure 5, which shows four selected bundles of field lines together with volume rendered surfaces of constant enstrophy (which coincide with sinking plumes, as can be seen from Figure 4). There is an obvious contrast between the tangled field lines in the upper region and the predominantly horizontal field lines below, which are only 


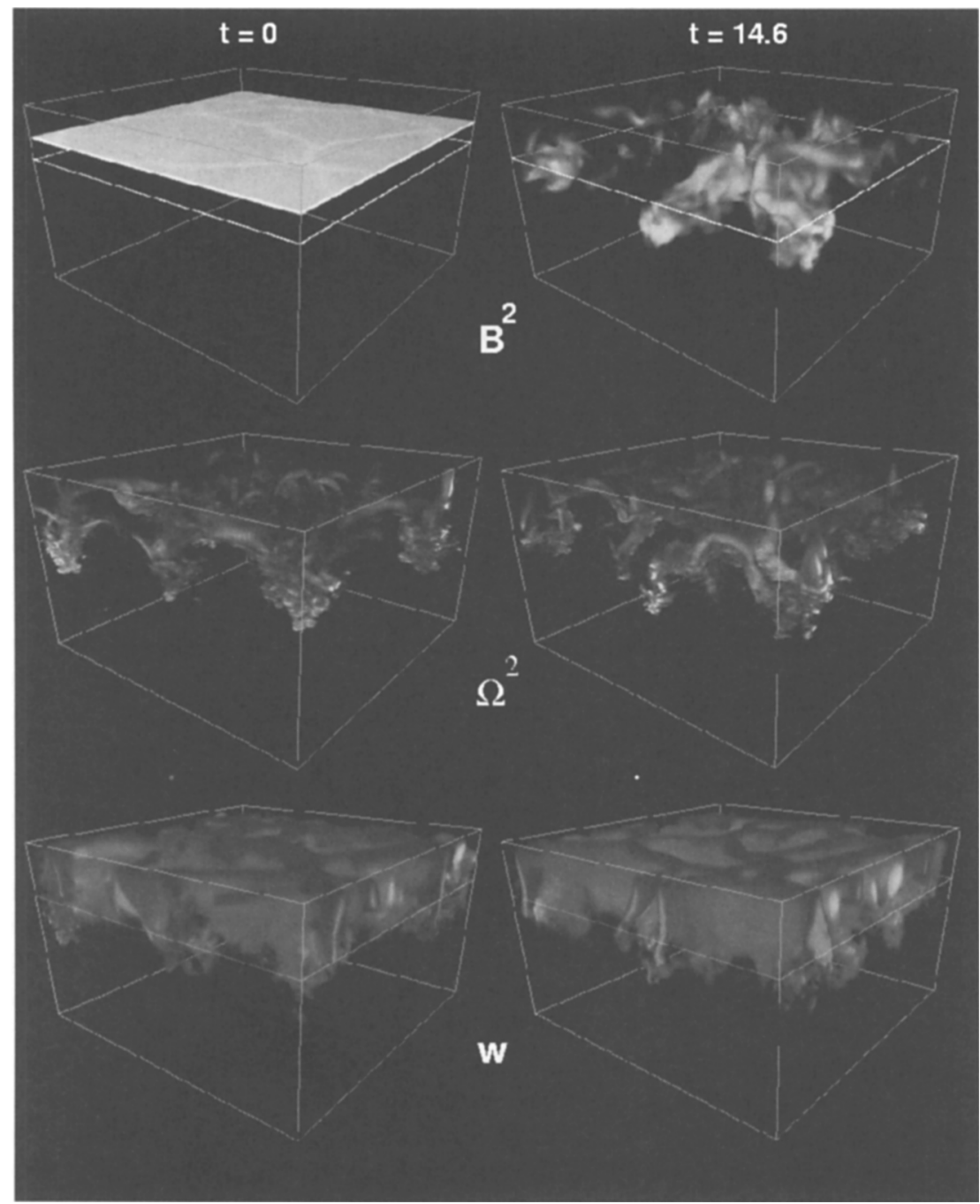

Figure 4. Downward pumping of magnetic flux by turbulent compressible convection. Left panels show the initial state, right panels show results at the end of the pumping phase. The volume rendering at the bottom shows the vertical velocity $w$, with red (blue) denoting up-(down-)ward motion. The enstrophy density $\Omega^{2}$ is represented in the middle and the magnetic energy $B^{2}$ is shown at the top. At time $t=0$ a thin layer of strong unidirectional field is inserted into an already convecting layer. By time $t=14.6$ (in appropriate units) magnetic flux has been pumped out of the upper layer and into the stably stratified region below. The role of the rapidly sinking plumes, which penetrate into the lower region, is clearly apparent. (After Thomas et al. 2002.) 


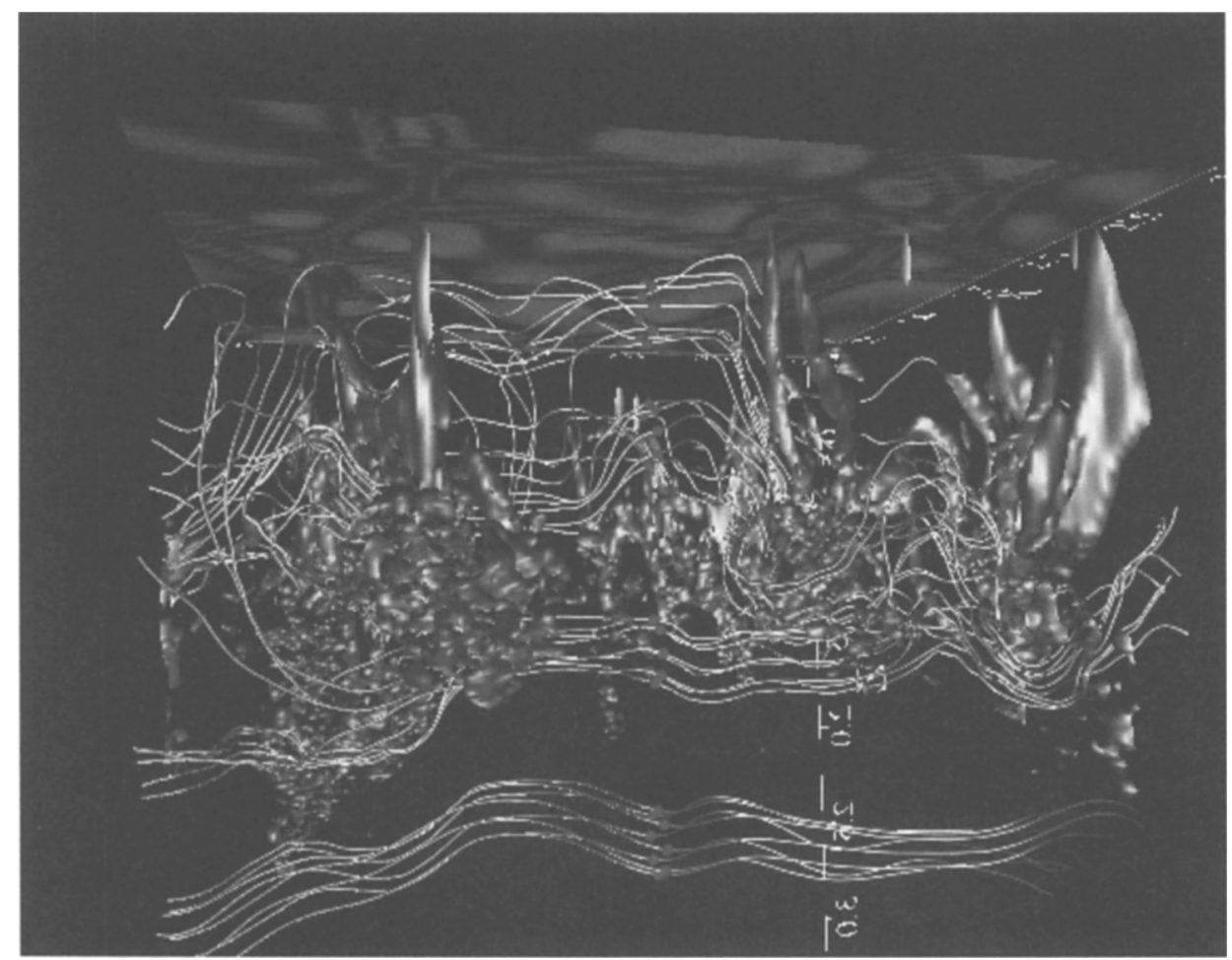

Figure 5. Perspective view of field lines when flux pumping has occurred. Red-blue shading indicates the vertical velocity just below the top, while the blue volume rendering shows surfaces of constant enstrophy, which indicate the locations of the rapidly sinking plumes. The instantaneous positions of four bundles of field lines, passing through the red beads, reveal the magnetic field. In the upper, unstable layer the field lines are strongly distorted but the original field direction is preserved when magnetic flux is pumped into the lower region. (After Thomas et al. 2002.) 
distorted by the penetrating plumes. (Note that these field lines only provide very local indications of variations in field strength.)

In a star like the Sun, magnetic flux is pumped downwards out of the convection zone and into the stably stratified radiative zone below. Since the interface is the site of the tachocline, where there is a strong shear in angular velocity, this pumping process is an important ingredient of the dynamo that maintains the star's magnetic field. The same process is also important in the solar photosphere. The shallow surface layer of vigorous granular and mesogranular convection is superposed on deeper, larger-scale supergranular motion which is relatively weak. So flux tubes can be held down in spite of being magnetically buoyant. This effect is believed to be responsible for the interlocking-comb structure of the magnetic fields in the penumbrae of sunspots (Thomas et al. 2002).

\subsection{Small-scale dynamos}

For my final topic, I shall take up the issue raised at the end of $\S 4.1$. What happens as the imposed magnetic field is further decreased until it becomes extremely small? The turbulent motion is chaotic, in the sense that the distance between neighbouring fluid elements increases exponentially, and this has two competing effects on the magnetic field. Field lines are stretched by the chaotic flow, thereby increasing the magnetic energy, but the transverse scale of variation is reduced, so enhancing dissipation. If the former process dominates, the motion will act as a small-scale dynamo, producing a disordered field. Cattaneo (1999) has demonstrated that turbulent convection in a Boussinesq fluid can indeed act as such a dynamo in the limit when $Q=0$ and there is no net imposed field, provided that the magnetic Reynolds number $R_{\mathrm{m}}$ is sufficiently large. In practice this requires that $R_{\mathrm{m}} \approx 1000$ (cf. Cattaneo 2002) and it is hard to attain this value in compressible calculations.

What is feasible, however, is to explore the effect of decreasing $Q$ in Boussinesq magnetoconvection. This has been done for a Rayleigh number $R=$ 500000 , with $\sigma=1$ and $\zeta=0.2$, in a wide box with $\lambda=10$ (Emonet, Cattaneo $\&$ Weiss 2001; Cattaneo, Emonet \& Weiss 2002). With this choice of parameters, convection sets in at a subcritical oscillatory bifurcation when $Q=370000$. Figures 6 and 7 show the behaviour found near the upper boundary as $Q$ is reduced over the range $50000 \geq Q \geq 3125$. For the strongest imposed field (case 4) there is an irregular pattern of small-scale cellular convection and magnetic flux is swept into a network that encloses the rising plumes as they impinge upon the rigid upper boundary. The field in this network has the same sign everywhere. Note that the up-down symmetry of Boussinesq convection ensures that there is a complementary pattern at the lower boundary, so the field within the layer is more complicated. The symmetry also ensures that there is no preferential pumping of magnetic flux. Reducing $Q$ allows the cells to widen, as shown for case 3 , but they are still enclosed by a network of unidirectional field. By case 2 it is apparent that the magnetic pattern has a larger scale than the thermal pattern and segments of oppositely directed field have appeared. With the lowest value of Q (case 1) a two-scale pattern has appeared (Cattaneo, Lenz $\&$ Weiss 2001). The magnetic fields surround clusters of convection cells and are strongest at the corners of this mesocellular network. By this stage the total 
crse 1

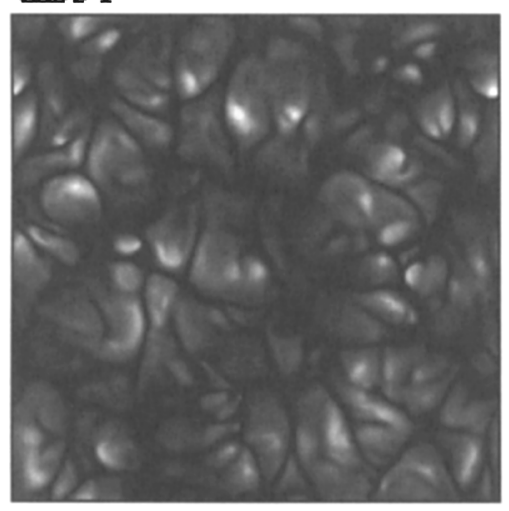

crse 3

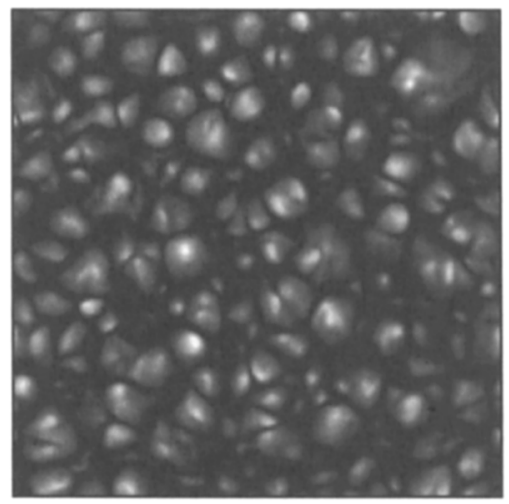

crse 2

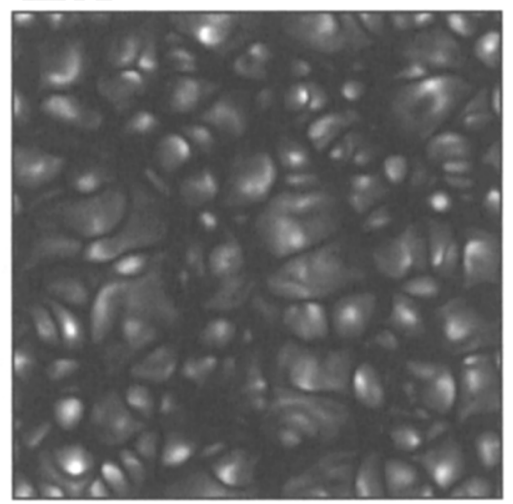

case 4

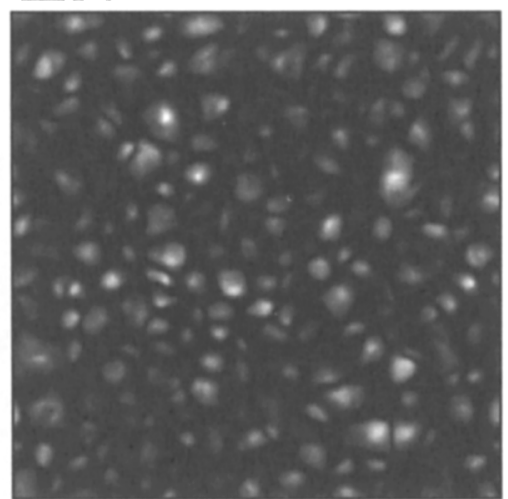

Figure 6. From magnetoconvection to small-scale dynamo action. Temperature fluctuations near the top of the layer for $Q=3125$ (case 1 ), $Q=12500$ (case 2), $Q=25000$ (case 3) and $Q=50000$ (case 4). Light regions are hot, dark regions are cool. (After Cattaneo et al. 2002.)

magnetic and kinetic energies are very nearly equal, and the overall pattern is very similar to that found when $Q=0$ and a weak small-scale field is allowed to grow until a statistically steady state has been reached (Cattaneo 1999).

It follows that there is a smooth transition from magnetoconvection to small-scale dynamo action. Moreover, there is every reason to expect that a similar transition could be found for compressible magnetoconvection with a sufficiently high value of $R_{\mathrm{m}}$. Owing to stratification, the behaviour of the dynamo will be complicated by downward pumping of magnetic flux. It seems likely that local dynamo action near the photosphere is responsible for producing the disordered small-scale fields that are constantly emerging to form a magnetic carpet in the Sun (see, for example, Simon, Title \& Weiss 2001). 
case 1

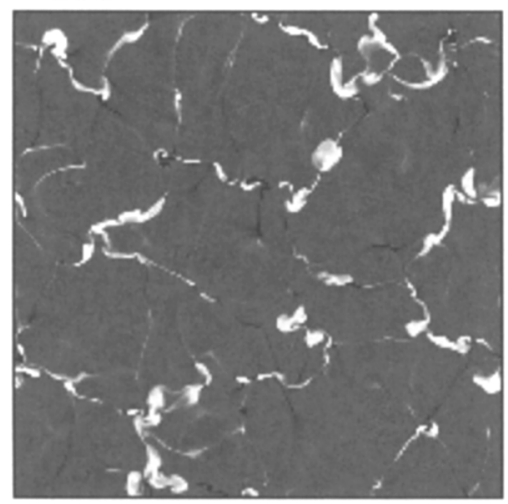

case 3

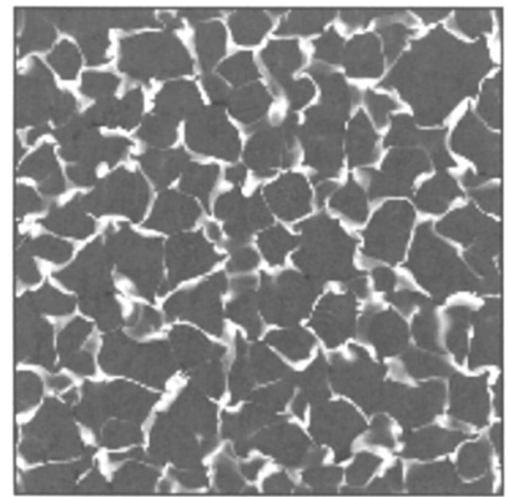

case 2

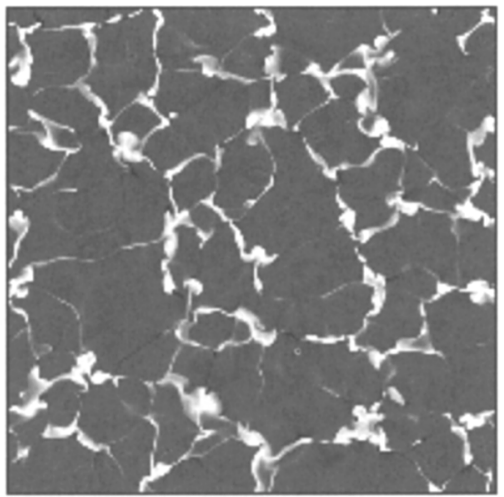

case 4

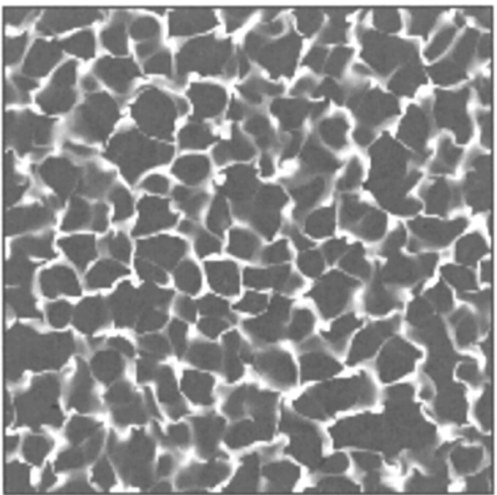

Figure 7. As Figure 6 but showing the magnetic field at the upper boundary, which is constrained to be vertical. In these images, white indicates strong fields parallel to the direction of the imposed field, black indicates oppositely directed fields and mid-grey corresponds to zero field. (After Cattaneo et al. 2002.)

\section{Looking ahead}

Although we have seen remarkable progress in computational modelling of stellar magnetoconvection there is still a long way to go. In the immediate future we can expect to carry out numerical experiments with higher values of $R$ and $R_{\mathrm{m}}$ as more powerful facilities become available. It will also be necessary to use wider boxes, with $\lambda \geq 12$, in order to study small-scale dynamo action in a stratified layer, where the rising plumes are broader. In addition, we shall be able to probe deeper into a compressible atmosphere and thus to gain a better idea of the structure of convection and the origin of the supergranular pattern that is so striking at the surface of the Sun. That will help in exploring the combined effects of granules, mesogranules and supergranules in pumping magnetic flux below the photosphere, which will in turn illuminate the structure 
of penumbral filaments in sunspots. Similarly, it will become possible to model three-dimensional convection in the presence of tilted magnetic fields and thus eventually to build up coherent models of sunspots and starspots.

In the longer term, what are needed are global, rather than local, calculations so that we can describe the interactions between convection and magnetic fields in the deep convection zones of late-type stars. Instead of modelling smallscale dynamo action, leading to disordered fields only, we want to represent the large-scale magnetic field that is responsible for cyclic activity in the Sun. It is rotation that produces macroscopic order out of microscopic chaos and so the first priority must be to establish how convection interacts with angular momentum in solar-type stars. Then we have to explain the differential rotation that has been revealed by helioseismology, together with the origin of the narrow tachocline. Only then will it be possible to produce a self-consistent model of the solar dynamo itself, such as exists already for the geodynamo. After that, we shall be ready to go on and provide more detailed models of dynamos in more active, rapidly rotating stars, and to explain why they exhibit polar spots.

Acknowledgments. The results described here owe much to Nic Brummell, Fausto Cattaneo, Thierry Emonet, Mike Proctor, Jack Thomas and Steve Tobias, and I thank them for many fruitful iand enjoyable discussions. I am also grateful to Joji Kuwabara and the late Yutaka Uchida for their help in producing Figure 5 .

\section{Discussion}

GRAY: Some time ago measurements of granulation layers were found to vary during the solar cycle. This seems to be at least qualitatively in agreement with your calculation. Has anyone, or have you, followed up on this point?

WEISS: High-resolution ground bound observation (for example, from the SVST on la Palma) show that the granulation in plage region is very different from that in the quiet Sun. The cells are indeed smaller, as expected from models of magnet-convection, and the patter is clearly abnormal. Thus there is indeed a variation in mean granulation size over the solar activity cycle, though the effect is not very large.

DRAVINS: The solar-cycle changes of granular sizes influences another important field of astronomy: the search for exoplanets. Spectral line profiles and wavelength shifts are somewhat different in magnetic granulation, producing an 11-year cycle in integrated sunlight of wavelength shifts of some 20 or $30 \mathrm{~m} / \mathrm{s}$. This is to compared with the $13 \mathrm{~m} / \mathrm{s}$ induced by Jupiter over 12 years, as the Sun is moving around their common center of mass. Thus, in order to distinguish real exoplanets with periods comparable to stellar activity cycles, from changes in magnetic convection, may require a good understanding of the latter.

STEPIEN: Have the computations of the flux pumping shown any approach to stationary situation (in a statistical sense) or the system evolved systematically till the end of the computations?

WEISS: The final state depends on the boundary conditions that are imposed. If no magnetic flux is allowed to escape, the system will eventually settle down 
to a statistically steady state. If magnetic flux can leak out through the upper then the field will eventually decay, but on a very long time-scale compared with that of pumping to occur.

STEPIEN: Do you think that sunspots (starspots) can be produced by the statistical fluctuations during magnetic convection computations or an additional mechanism of flux concentration is needed?

WEISS: No, such large magnetic features can only be produced by some global process, presumably a large-scale field generated by a dynamo located at the base of the convection zone. The magnetic flux that emerges in ephemeral active regions to form the 'magnetic carpet' is probably produced by small-scale dynamo action somewhere below the photosphere.

\section{References}

Arter, W. 1983, JFM, 132, 25

Arter, W. 1985, GAFD, 25, 311

Biermann, L. 1941, Vierteljahrsschr. Astr. Ges., 76, 194

Brandenburg, A., Jennings, R.L., Nordlund, Å, Rieutord, M., Stein, R.F. \& Tuominen, I. 1996, JFM, 306, 325

Cattaneo, F. 1999, ApJ, 515, L39

Cattaneo, F. 2002, in IAU Symp. 210, Modelling of Stellar Atmospheres, N. E. Piskunov, W. W. Weiss \& D.F. Gray. San Francisco: ASP, these proceedings

Cattaneo, F., Emonet, T. \& Weiss, N.O. 2002, ApJ, to be submitted

Cattaneo, F., Lenz, D. \& Weiss, N.O. 2001, ApJ, 563, L91

Chandrasekhar, S. 1952, Phil. Mag. (7), 43, 501

Chandrasekhar, S. 1961, Hydrodynamic and Hydromagnetic Stability, Oxford: Clarendon Press

Cowling, T.G. 1953, in The Sun, G.P. Kuiper, Chicago: U. Chicago Press, 532

Cowling, T.G. 1957, Magnetohydrodynamics. New York: Interscience

Danielson, R.E. 1961, ApJ, 134, 275

Dorch, S.B.F. \& Nordlund, Å, 2001, A\&A, 365, 562

Drobyshevski, E.M. \& Yuferev, V.S. 1974, JFM, 65, 33

Emonet, T., Cattaneo, F. \& Weiss, N.O. 2001, in Dynamo and Dynamics: a Mathematical Challenge, P. Chossat, G. Dangelmayr \& J. Oprea.. Dordrecht: Kluwer, 173

Galloway, D.G. \& Proctor, M.R.E. 1983, GAFD, 24, 109

Guckenheimer, J. \& Holmes, P. 1986, Nonlinear Oscillations, Dynamical Systems and Bifurcations of Vector Fields. New York: Springer

Hughes, D.W. \& Proctor, M.R.E. 1988, ARFM, 280, 227

Hurlburt, N.E. \& Toomre, J. 1988, ApJ, 327, 920

Hurlburt, N.E., Proctor, M.R.E., Weiss, N.O. \& Brownjohn, D.P. 1989, JFM, 207,587 
Hurlburt, N.E., Matthews, P.C. \& Rucklidge, A.M. 2000, Sol. Phys., 192, 109

Knobloch, E. \& Proctor, M.R.E. 1981, JFM, 108, 291

Knobloch, E., Weiss, N.O. \& DaCosta, L.N. 1981, JFM, 113, 153

Matthews, P.C., Proctor, M.R.E., Rucklidge, A.M. \& Weiss, N.O. 1993, Phys. Lett. A, 183, 69

Mestel, L. 2001, Stellar Magnetism. Oxford: Clarendon Press

Nordlund, A, 1983, in IAU Symp. 102, Solar and Stellar Magnetic Fields: Origins and Coronal Effects, J.O. Stenflo. Dordrecht: Reidel, 79

Nordlund, $\AA, 1984$, in The Hydromagnetics of the Sun, Noordwijk: ESA SP-220, 37

Nordlund, A. \& Stein, R.F. 1989, in Solar and Stellar Granulation, R.J. Rutten $\&$ G. Severino. Dordrecht: Kluwer, 453

Nordlund, Å. \& Stein, R.F. 1990, in IAU Symp. 138, Solar Photosphere: Structure, Convection and Magnetic Fields, J.O. Stenflo. Dordrecht: Kluwer, 191

Nordlund, Å, Brandenburg, A., Jennings, R.L., Rieutord, M., Ruokalainen, J., Stein, R.F. \& Tuominen, I. 1992, ApJ, 392, 647

Parker, E. N. 1979, Cosmical Magnetic Fields: their Origin and Activity. Oxford: Clarendon Press

Proctor, M.R.E. \& Weiss, N.O. 1982, Rep. Prog. Phys. 45, 1317

Proctor, M.R.E., Weiss, N.O., Brownjohn, D.P. \& Hurlburt, N.E. 1994, JFM, 280,227

Rädler, K.-H. 1968, Z. Naturforsch. 23, 1851

Rucklidge, A.M. 1992, JFM, 237, 209

Rucklidge, A.M. 1994, Nonlinearity, 7, 1565

Rucklidge, A.M., Weiss, N.O., Brownjohn, D.P., Matthews, P.C. \& Proctor, M.R.E. 2000, JFM, 419, 283

Simon, G.W., Title, A. \& Weiss, N.O. 2001, ApJ, 561, 427

Spruit, H.C., Nordlund, Å. \& Title, A.M. 1990, ARA\&A, 28, 263

Stein, R.F. \& Nordlund, Å. 1998. ApJ, 499, 914

Stein, R.F. \& Nordlund, A. 2002, in IAU Symp. 210, Modelling of Stellar Atmospheres, N. E. Piskunov, W. W. Weiss \& D.F. Gray. San Francisco: ASP, these proceedings

Stein, R.F., Bercik, D. \& Nordlund, Å. 2002. Nuovo Cimento, in press

Tao, L.L., Proctor, M.R.E. \& Weiss, N.O. 1998a, MNRAS, 300, 907

Tao, L.L., Weiss, N.O., Brownjohn, D.P. \& Proctor, M.R.E. 1998b, ApJ, 496, L39

Thomas, J.H. \& Weiss, N.O. 1992, in Sunspots: Theory and Observations, J.H. Thomas \& N.O. Weiss. Dordrecht: Kluwer, 3

Thomas, J.H., Weiss, N.O., Tobias, S.M. \& Brummell, N.H. 2002, Nature, in press

Thompson, W.B. 1951, Phil. Mag. (7), 42, 1417 
Thomson, J.J. 1893, Recent Researches in Electricity and Magnetism. Oxford: Clarendon Press

Tobias, S.M., Brummell, N.H., Clune, T.L. \& Toomre, J. 1998, ApJ, 502, L177

Tobias, S.M., Brummell, N.H., Clune, T.L. \& Toomre, J. 1998, ApJ, 549, 1183

Veronis, G. 1959, JFM, 5, 401

Vögler, A. \& Schüssler, M. 2002, in IAU Symp. 210, Modelling of Stellar Atmospheres, N. E. Piskunov, W. W. Weiss \& D.F. Gray. San Francisco: ASP, these proceedings

Walén, C. 1949, On the Vibratory Motion of the Sun. Stockholm: Henrik Lindstahl.

Weiss, N.O. 1966, Proc. Roy. Soc. A, 293, 310

Weiss, N.O. 1981a, JFM 108, 247

Weiss, N.O. 1981b, JFM 108, 273

Weiss, N.O. 1991, GAFD, 62, 229

Weiss, N.O., Brownjohn, D.P., Hurlburt, N.E. \& Proctor, M.R.E. 1990, MNRAS, 245, 434

Weiss, N.O., Brownjohn, D.P., Matthews, P.C. \& Proctor, M.R.E. 1996, MNRAS, 283, 1153

Weiss, N.O., Proctor, M.R.E. \& Brownjohn, D.P. 2002, MNRAS, in press

Zeldovich, Ya.B. 1956, JETP, 31, 154 (trans. 4, 460, 1957)

Zeldovich, Ya.B., Ruzmaikin, A.A. \& Sokoloff, D.D. 1983, Magnetic Fields in Astrophysics. New York: Gordon \& Breach 